J. Lake Sci.(湖泊科学), 2018, 30(4): 907-915

DOI 10. 18307/2018. 0404

(c) 2018 by Journal of Lake Sciences

\title{
蓝藻水华暴发和衰亡对太湖有色可溶性有机物的影响”
}

\author{
乔显琦, 江海洋, 李 星, 王志刚, 陈小锋** \\ (扬州大学环境科学与工程学院,扬州 225127)
}

\begin{abstract}
摘 要: 利用平行因子分析 (PARAFAC) 技术对藻华暴发季节太湖梅梁湾和开敞区水样中有色可溶性有机物 (CDOM) 的 三维苂光光谱进行分析, 获得代表类酪氨酸、类色氨酸和类腐殖酸等 3 种苂光组分, 这 3 个组分的苂光得分值均与叶绿素 $\mathrm{a}$ 浓度呈极显著正相关, 其中类腐殖酸物质苂光得分值占总分值的比例也与叶绿素 a 浓度极显著正相关, 由此可以推测, 蓝藻水华可能是太湖 CDOM 的一个重要来源, 并极大地影响了湖泊 CDOM 的组成结构. 为进一步探索蓝藻水华对湖泊 CDOM 的影响, 于太湖梅梁湾湖岸进行藻华暴发和衰亡模拟试验. PARAFAC 结果显示, 模拟试验水样 CDOM 的三维苂光 图谱同样含有 3 个组分, 其中类酪氨酸和类色氨酸菼光峰位置与湖泊水样基本相同, 而类腐殖酸物质苂光峰则有一定程 度的蓝移, 表明培养试验中有新的内源类腐殖酸物质产生. 利用 PARAFAC 模型中的得分值计算后发现, 初始叶绿素 a 浓 度越高, 类腐殖酸物质所占比例越大, 而实验结束时添加了高浓度和中等浓度藻的 2 个处理中类腐殖酸物质对苂光的贡 献率也有显著提高. 因此, 藻华的长期暴发可以显著改变湖泊 CDOM 的组成,导致类腐殖酸物质比例提高.
\end{abstract}

关键词: 蓝藻水华;有色可溶性有机物;组成结构;平行因子分析法;光谱学;太湖;梅梁湾

\section{Impacts of cyanobacterial blooms outbreak and decline on chromophoric dissolved organic matter in Lake Taihu}

\author{
QIAO Yuqi, JIANG Haiyang, LI Xing, WANG Zhigang \& CHEN Xiaofeng ** \\ (School of Environmental Science and Engineering, Yangzhou University, Yangzhou 225127, P.R.China)
}

Abstract: Parallel Factor Analysis (PARAFAC) was applied to assess the composition of chromophoric dissolved organic matter ( CDOM) from the excitation-emission matrix ( EEM) fluorescence spectra of the water samples collected from Meiliang Bay and the open area in Lake Taihu during a cyanobacterial bloom event, and three fluorescent components were identified: a tyrosine-like component, a tryptophan-like component and a humic-like component. Correlation analysis showed that the concentrations of chlorophyll-a(Chl.a) were significantly correlated with the fluorescence intensities of the three components, respectively, as well as the proportion of humic-like to total fluorescence intensities, suggesting that cyanobacteria might be an important source of CDOM and can greatly change the composition of CDOM in Lake Taihu. For further investigation, a simulation experiment was performed on the shore of Meiliang Bay. PARAFAC model showed that the number of components and the spectral characteristics of CDOM EEM data from the simulation experiment are similar in terms of the number of fluorescence peaks and their position to previously identified components in the water samples from Lake Taihu, except that the fluorescence peaks of humic acids showed a certain degree of blue shift, which suggested that endogenous humic substances were produced during the simulation experiment. It was found that the higher initial concentrations of Chl.a, the greater contribution of humic acids to total CDOM fluorescence intensity derived by the PARAFAC model. Moreover, at the end of the experiment, the ratios of humic substances to total fluorescent substances increased significantly in the two treatments with relatively high concentrations of cyanobacteria. Thus, the long-term outbreak of cyanobacterial blooms can significantly alter the composition of the CDOM in lakes, resulting in an increase of the proportion of humiclike substances.

Keywords: Cyanobacterial bloom; chromophoric dissolved organic matter; composition and structure; PARAFAC; spectroscopy; Lake Taihu; Meiliang Bay

* 国家自然科学基金项目 (41373076) 资助. 2017-10-11 收稿; 2017-12-25 收修改稿. 乔显琦 (1993 ),女,硕士研 究生; E-mail: 553750279@qq.com.

** 通信作者; E-mail: xfchen@yzu.edu.cn. 
湖泊有色可溶性有机物 (CDOM) 是溶解性有机物中的光活性部分, 一般来源于外源腐殖质的输人和内 源水生植物的降解 ${ }^{[1-3]}$. CDOM 在湖泊生态系统中具有重要作用. 首先, CDOM 可以吸收紫外和可见波段范 围内的光, 一方面造成了水下光合有效辐射的衰减, 从而在一定程度上抑制了浮游植物和沉水植物的生

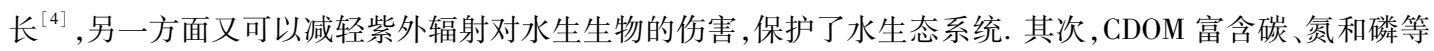
湖泊生源要素, 在光化学作用下可以释放出易被微生物和水生植物利用的低分子量化合物, 从而对湖泊富 营养化进程和碳循环等有着巨大影响 ${ }^{[5-6]}$.

CDOM 化学成分复杂,一般认为其主要由类腐殖质和类蛋白等一系列物质组成 ${ }^{[7]}$. 研究发现, 类腐殖质 分子上某些官能团与水体中的痕量金属元素有很强的结合能力, 从而在重金属的迁移和归趋过程中起着重 要作用 ${ }^{[8]}$, 而类腐殖质中类腐殖酸与类富里酸的相对比例又极大地影响着其光吸收特性 ${ }^{[9]}$. 此外, CDOM 中 所含有的类蛋白等生物可利用组分与异养细菌的新陈代谢有较强的正相关性 ${ }^{[10]}$, 其含量的变化可以显著改 变水体中微生物的群落结构 ${ }^{[11]}$. 因此, 研究 CDOM 组分的变化情况, 对于评估 CDOM 在水生态系统中的作 用有着重要的意义.

过去的 20 30 年内, 由于流域内和周围城市大量氮和磷营养盐的输人, 太湖面临着严重的富营养化问 题, 并频繁暴发蓝藻水华. 藻华衰亡对湖泊 CDOM 的贡献已有较多研究 ${ }^{[12-13]}$, 但针对蓝藻暴发和衰亡过程中 湖泊 CDOM 的组成变化的研究甚少. 平行因子分析 (PARAFAC) 技术可以有效地将 CDOM 三维荧光光谱分 解成各个独立的苂光组分, 并已被广泛应用于水体中 CDOM 苂光成分的动态变化研究 ${ }^{[3,14-15]}$, 因此本研究采 用三维荧光光谱和平行因子分析法, 对湖泊实地调查和模拟试验的样品进行分析, 并结合水质理化参数的 测定,考察蓝藻水华暴发和衰亡对湖泊 CDOM 的影响.

\section{1 材料与方法}

\section{1 研究区域和采样位点}

太湖 ( $30^{\circ} 56^{\prime} \sim 31^{\circ} 33^{\prime} \mathrm{N}, 119^{\circ} 54^{\prime} \sim 120^{\circ} 36^{\prime} \mathrm{E}$ ), 位于长江三角洲地区, 是中国五大淡水湖之一( 面积为 $2338 \mathrm{~km}^{2}$ ), 同时也是典型的富营养化湖泊 ${ }^{[16]}$. 过去 30 年来, 蓝藻水华已经成为严重的环境问题 ${ }^{[17]}$. 梅梁湾 位于太湖西北部, 面积 $144 \mathrm{~km}^{2}$, 受东南季风的影响, 每年 5-9 月均有大量的蓝藻堆积于湾内湖面上, 使得 其叶绿素 $\mathrm{a}$ 平均浓度可达到近 $50 \mu \mathrm{g} / \mathrm{L}^{[18]}$.

为考察藻华暴发对湖泊 CDOM 的影响, 于藻华暴发季节 (2016 年 8 月) 进行湖泊采样调查, 各位点分布 如图 1 所示, 其中位点 $1^{\#} \sim 3^{\#}$ 分别为武进港、直湖港和梁溪河人湖河口, 位点 $8^{\#}$ 和 $9^{\#}$ 为梅梁湾湾口, 而位点 12 代表湖心位置. 受主导风向的影响, 实验期间大量蓝藻堆积在位点 $2^{\#}$ 处, 因此模拟实验所使用的蓝藻样 品在此处采集. 中国科学院南京地理与湖泊研究所太湖湖泊生态系统研究站 (TLLER) 位于梅梁湾东侧近湾 口处,本次野外模拟实验在该研究站内进行.

\section{2 湖泊调查}

采集太湖梅梁湾、开敞区等 12 个位点 (图 1) 的表层水样 (水面下 $30 \sim 50 \mathrm{~cm}$ ), 同时测定水温、 $\mathrm{pH}$ 和溶解 氧 (DO) 等指标, 所采集样品置于 $4^{\circ} \mathrm{C}$ 冷藏箱保存, 回实验室后立即测定叶绿素 $\mathrm{a}(\mathrm{Chl} . \mathrm{a})$ 、总氮 (TN)、总磷 ( TP )、硝态氮 $\left(\mathrm{NO}_{3}^{-}-\mathrm{N}\right)$ 和铵态氮 $\left(\mathrm{NH}_{4}^{+}-\mathrm{N}\right)$ 等水质参数, 并进行三维苂光扫描.

\section{3 模拟试验}

实验前一天于位点 $2^{\#}$ 采集蓝藻, 藻种鉴定结果表明, 铜绿微囊藻为优势种. 将采集的蓝藻分别加人到含 $400 \mathrm{~L}$ 湖水 (直接从湖中洜人) 的塑料容器中, 使得 Chl.a 初始浓度分别约为 $150 、 100$ 和 $50 \mu \mathrm{g} / \mathrm{L}$, 每个浓度设 3 个平行. 太湖蓝藻在夏季处于氮限制状态 ${ }^{[18]}$, 为观察澡类生长和衰亡两个阶段对湖泊 CDOM 的影响, 在实 验初始和中间各添加 1 次氯化铵. 实验过程中, 每天上午 $6: 00$ 和下午 $6: 30$ 测定水温、 $\mathrm{pH}$ 和 DO 浓度等指 标, 在检测完这些理化性质之后, 充分搅拌后取样, 样品在 $\mathrm{Chl} . \mathrm{a} 、 \mathrm{NO}_{3}^{-}-\mathrm{N}$ 和 $\mathrm{NH}_{4}^{+}-\mathrm{N}$ 浓度等水质参数测定前置 于 $4^{\circ} \mathrm{C}$ 冰箱中保存.

\section{4 理化性质分析和光谱扫描}

水温、 $\mathrm{pH}$ 和 DO 等指标使用 YSI 6600 多功能水质仪现场测定, Chl. a 、 TN、TP、 $\mathrm{NO}_{3}^{-}-\mathrm{N}$ 和 $\mathrm{NH}_{4}^{+}-\mathrm{N}$ 浓度的测 定按照国家环境保护总局标准分析方法 ${ }^{[19]}$ 进行. 水样经 $0.7 \mu \mathrm{m}$ 预灼烧的玻璃纤维滤膜过滤后, 采用日立 


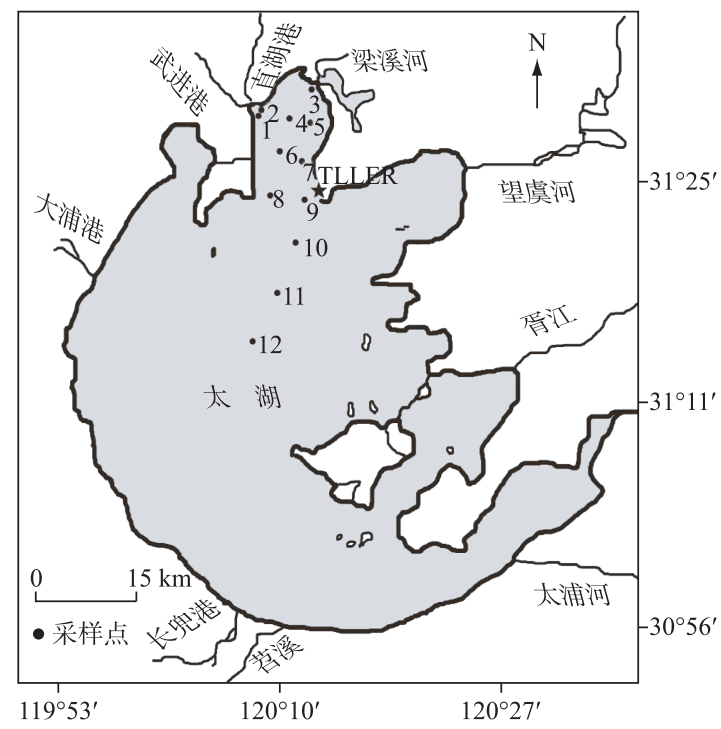

图 1 研究区域位置和采样位点分布

Fig.1 Location of studied area and sampling sites

F-7000 苂光分光光度计进行 CDOM 三维荧光光谱扫描, 激发波长 $(\mathrm{Ex})$ 和发射波长 $(\mathrm{Em})$ 扫描范围分别为 $200 \sim 450$ 和 $250 \sim 600 \mathrm{~nm}$, 扫描步长 $5 \mathrm{~nm}$, 狭缝宽度 $5 \mathrm{~nm}$, 扫描速度 $12000 \mathrm{~nm} / \mathrm{min}$.

\section{5 数据处理}

为消除水的拉曼散射和瑞利散射的影响, 将获得的苂光光谱矩阵数据减去 Milli-Q 超纯水三维苂光光谱 数据, 并将图谱中 $\mathrm{Ex} \geqslant \mathrm{Em} 、 2 \times \mathrm{Ex} \leqslant \mathrm{Em}$ 及 $\mathrm{Ex}=\mathrm{Em} \pm 10 \sim 15 \mathrm{~nm}$ 等区域中的数据以 0 代替. 为便于不同仪器间 的比较, 对苂光强度进行定标处理, 即将 $0.01 \mathrm{mg} / \mathrm{L}$ 硫酸奎宁的稀硫酸溶液在 $\mathrm{Ex} / \mathrm{Em}=350 \mathrm{~nm} / 450 \mathrm{~nm}$ 下的 苂光强度定义为 1 个归一化苂光单位, 单位为 $\mathrm{QSU}^{[12]}$.

采用 SPSS 18.0 软件进行相关性分析和差异性检验, $P<0.05$ 说明相关性或差异性显著. 采用 Sigmaplot 12.5 和 Matlab R2011a 软件进行作图.

\section{2 结果与讨论}

\section{1 湖泊调查}

2.1.1 理化性质 湖泊采样在下午 $2: 00-4: 00$ 进行, 天气多云且风力小于 3 级, 因此大量蓝藻上浮并堆积在 梅梁湾湖面上. 各采样点中河口位置 (位点 $1^{\#} \sim 3^{\#}$ ) 的蓝藻最为密集, 其平均 Chl.a 浓度达到 $283.20 \mu \mathrm{g} / \mathrm{L}$, 梅梁 湾 (位点 $4^{\#} \sim 9^{\#}$ ) 次之, 平均 Chl.a 浓度为 $82.00 \mu \mathrm{g} / \mathrm{L}$, 开敞区 (位点 $10^{\#} \sim 12^{\#}$ ) 最低, 只有 $23.00 \mu \mathrm{g} / \mathrm{L}($ 表 1 ). 与 Chl.a 浓度相对应, 河口 TN 和 TP 浓度最高, 其中位点 $1^{\#}$ 的 TN 和 TP 浓度分别达到 10.2 和 $0.22 \mathrm{mg} / \mathrm{L}$, 开敞 区相对较低,但 TN 和 TP 浓度平均值也分别达到 2.45 和 $0.07 \mathrm{mg} / \mathrm{L}$. 尽管 TN 浓度很高, 无机氮浓度却相对 较低, 3 个区域的 $\mathrm{NH}_{4}^{+}-\mathrm{N}$ 浓度均只有 $0.08 \sim 0.09 \mathrm{mg} / \mathrm{L}$. 藻类的光合作用消耗了水中 $\mathrm{HCO}_{3}^{-}\left(\mathrm{CO}_{2}\right)$, 因此所有 采样位点的 $\mathrm{pH}$ 均在 11 左右. 采样当天梅梁湾湖面的蓝藻大多呈黄绿色, 表明有部分蓝藻已开始衰亡, 藻的 衰亡消耗了水体中的氧, 使得梅梁湾水体的 DO 水平显著低于河口和开敞区, 其中位点 $8^{\#}$ 的 DO 浓度仅有 $4.17 \mathrm{mg} / \mathrm{L}$.

2.1.2 湖泊 CDOM 三维荧光图谱 为考察太湖梅梁湾和开敞区 CDOM 的分布特征和组成情况, 对样品进行 了三维苂光扫描 (图 2), 可以看出, 苂光峰有 4 个, 包括类富里酸物质和类腐殖酸物质的菼光峰 $\mathrm{A}(\mathrm{Ex} / \mathrm{Em}=$ $255 \mathrm{~nm} / 455 \mathrm{~nm})$ 和 $\mathrm{C}(\mathrm{Ex} / \mathrm{Em}=360 \mathrm{~nm} / 455 \mathrm{~nm})$, 和类色氨酸和酪氨酸物质的苂光峰 $B(E x / E m=280 \mathrm{~nm} /$ $320 \mathrm{~nm})$ 和 $\mathrm{D}(\mathrm{Ex} / \mathrm{Em}=230 \mathrm{~nm} / 340 \mathrm{~nm})^{[20]}$. 其中, 类腐殖酸物质 ( $\mathrm{C}$ 峰) 在武进港人湖河口 (位点 $1^{\#}$ ) 有较强 
的荧光峰, 但是随着采样位点向湖心方向移动, 其强度逐渐减小, 到湖心 (位点 $12^{*}$ ) 时, C 峰已经消失.

表 1 湖泊基本水质参数

Tab.1 Values of water parameters in original water from Lake Taihu

\begin{tabular}{cccc}
\hline 水质参数 & 河口 $\left(\right.$ 位点 $\left.1^{\#} \sim 3^{\#}\right)$ & 梅梁湾 $\left(\right.$ 位点 $\left.4^{\#} \sim 9^{\#}\right)$ & 开敞区 $\left(\right.$ 位点 $\left.10^{\#} \sim 12^{\#}\right)$ \\
\hline $\mathrm{DO} /(\mathrm{mg} / \mathrm{L})$ & $9.57 \pm 2.11$ & $6.00 \pm 1.10$ & $11.87 \pm 1.57$ \\
$\mathrm{pH}$ & $11.26 \pm 0.49$ & $11.16 \pm 0.29$ & $10.98 \pm 0.37$ \\
$\mathrm{NH}_{4}^{+}-\mathrm{N} /(\mathrm{mg} / \mathrm{L})$ & $0.08 \pm 0.02$ & $0.09 \pm 0.03$ & $0.09 \pm 0.03$ \\
$\mathrm{NO}_{3}^{-}-\mathrm{N} /(\mathrm{mg} / \mathrm{L})$ & $0.74 \pm 0.18$ & $0.54 \pm 0.05$ & $0.43 \pm 0.04$ \\
$\mathrm{Chl} \cdot \mathrm{a} /(\mu \mathrm{g} / \mathrm{L})$ & $283.20 \pm 174.80$ & $82.00 \pm 35.80$ & $23.00 \pm 4.90$ \\
$\mathrm{TN} /(\mathrm{mg} / \mathrm{L})$ & $7.43 \pm 2.58$ & $4.38 \pm 0.45$ & $2.45 \pm 0.40$ \\
$\mathrm{TP} /(\mathrm{mg} / \mathrm{L})$ & $0.22 \pm 0.08$ & $0.12 \pm 0.04$ & $0.07 \pm 0.03$ \\
\hline
\end{tabular}
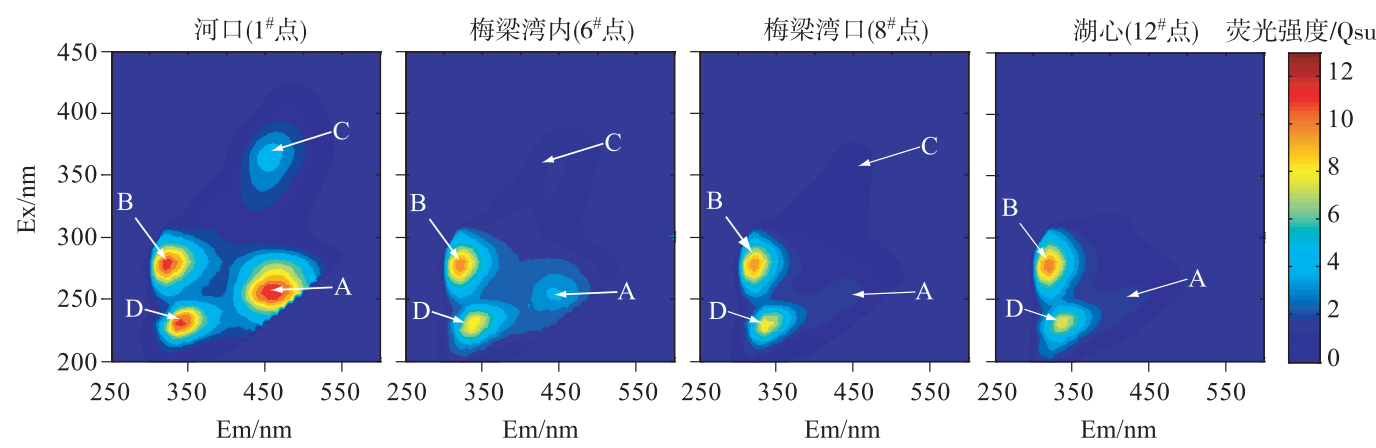

图 2 不同采样点表层水样 CDOM 的三维苂光光谱

Fig.2 EEM Spectra of the CDOM at different sampling sites

表 2 太湖表层水样各苂光峰强度与 Chl.a 浓度的相关性分析

Tab. 2 Correlations between different fluorecence intensities and Chl.a concentration

\begin{tabular}{cccccc}
\hline & A & B & C & D & Chl.a \\
\hline A & 1 & & & & \\
B & $0.842^{* * *}$ & 1 & & & \\
C & $0.999^{* *}$ & $0.824^{* *}$ & 1 & & \\
D & $0.906^{* *}$ & $0.920^{* *}$ & $0.895^{* *}$ & 1 & \\
Chl.a & $0.995^{* *}$ & $0.819^{* * *}$ & $0.995^{\text {*** }}$ & $0.868^{* *}$ & 1 \\
\hline
\end{tabular}

***显著性水平为 $P<0.01$ (双尾检验).
相关性分析经常被用来判定湖泊中不同苂光组 分来源的差异 ${ }^{[12]}$. 一般来讲, 如果类蛋白物质与类腐 殖酸苂光峰之间具有相关性, 那么可以认为它们具 有相同的来源. 因此, 太湖表层水样中类腐殖酸苂光 峰 $\mathrm{A}$ 和 $\mathrm{C}$ 与类蛋白苂光峰 $\mathrm{B}$ 和 $\mathrm{D}$ 的菼光强度呈显著 正相关 (表 $2, P<0.01$ ), 表明这两类物质可能具有相 似的来源 ${ }^{[12]}$, 而这些苂光峰的强度与 Chl.a 浓度又呈 现显著正相关 (表 $2, P<0.01$ ), 说明藻华的暴发对湖 泊水体类蛋白物质和类腐殖酸物质的产生均具有重 要作用.

利用平行因子分析法 (PARAFAC) 对湖泊水样 的三维苂光光谱进行分析, 当因子数大于 3 时, 核一

致函数值小于 $50 \%$, 且残差降低甚少, 因此建立因子数为 3 的 PARAFAC 模型, 获得 3 种苂光组分 (图 3). 组分 1 在最大发射波长 $320 \mathrm{~nm}$ 处有 2 个最大的激发波长 $(225 \mathrm{~nm}$ 和 $275 \mathrm{~nm}$ ), 其发射波长与图 2 中的 $\mathrm{B}$ 峰 位置相同, 表征的是类酪氨酸荧光物质. 组分 2 也有 2 个最大的激发波长, 分别在 235 和 $300 \mathrm{~nm}$ 处, 最大发 射波长为 $350 \mathrm{~nm}$, 与图 2 中的 $\mathrm{D}$ 峰位置相似, 代表类色氨酸物质, 这 2 个组分都被认为是由水里的生物死亡 降解所产生. 组分 3 的 2 个最大激发波长和 1 个最大发射波长分别为 $260 、 355$ 和 $460 \mathrm{~nm}$, 与图 2 中 $\mathrm{A}$ 峰和 $\mathrm{C}$ 峰相似, 被认为可能是由陆源输人的类腐殖质苂光物质 ${ }^{[12]}$. 从解析出的 3 个苂光峰图谱可以看出, 3 个组分 中类酪氨酸物质的苂光强度最高, 类腐殖酸物质最低.

根据 PARAFAC 分析结果中的得分值计算 3 个组分对各样品苂光强度的贡献率, 结果发现, 类腐殖酸苂 


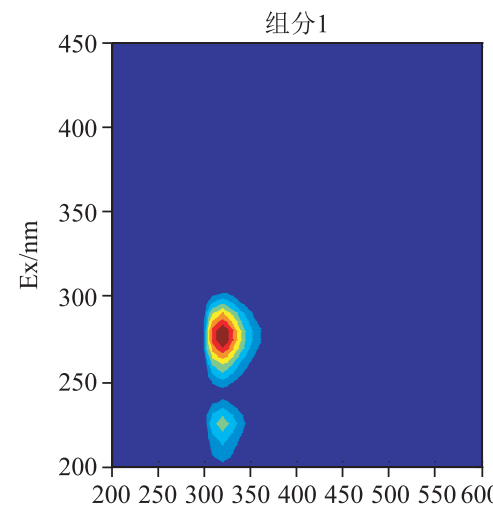

$\mathrm{Em} / \mathrm{nm}$

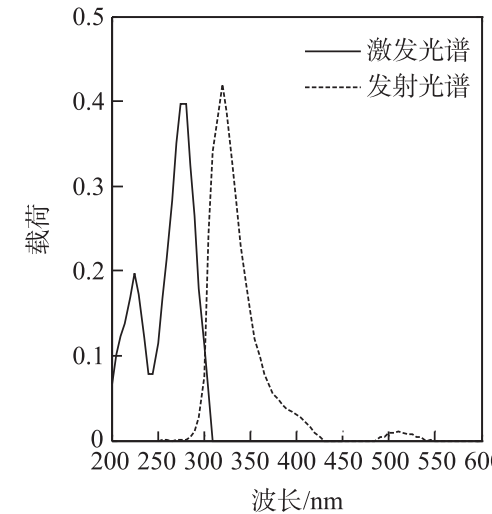

图 3 PARAFAC 模型解析出的 3 个组分
组分2

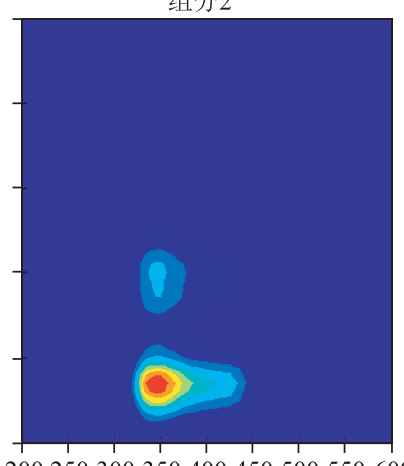

$\mathrm{Em} / \mathrm{nm}$

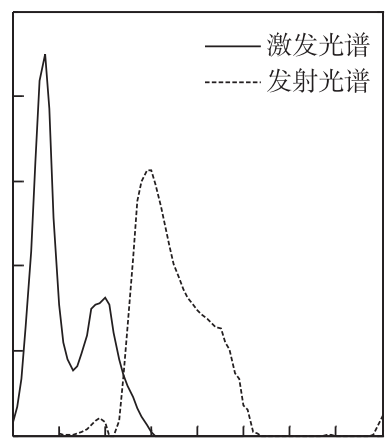

波长 $/ \mathrm{nm}$
组分3

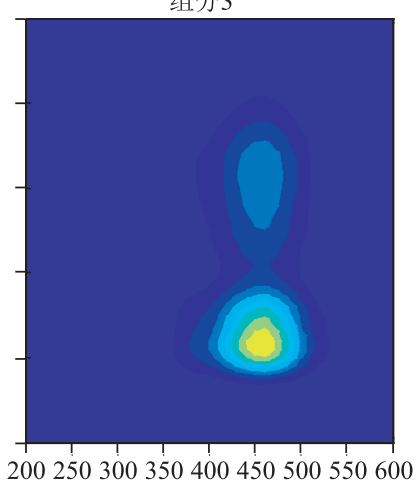

$\mathrm{Em} / \mathrm{nm}$

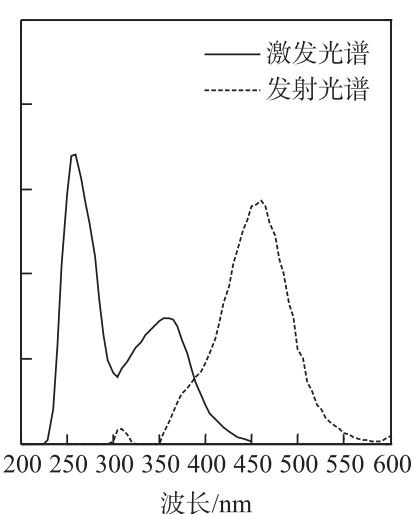

波长 $/ \mathrm{nm}$

Fig.3 The PARAFAC model output showing fluorescence signatures of three components identified

光所占比例基本呈现从梅梁湾至湖心方向逐渐下降的趋势, 该现象可能是河道输人的类腐殖酸物质逐步被 稀释造成. 但是前文分析结果表明藻华的暴发对湖泊类腐殖酸物质的产生具有重要作用,而类腐殖酸苂光 所占比例与叶绿素 a 浓度又呈极显著正相关 $\left(r^{2}=0.906, P<10^{-5}\right)$, 因此可以推测, 除稀释作用外, 藻华暴发 程度的差异可能是类腐殖酸菼光的比例从梅梁湾至湖心方向逐渐下降的另一个重要原因.

\section{2 藻华暴发和衰亡模拟试验}

为进一步探索藻华对湖泊 CDOM 的影响, 于太湖湖岸进行藻华暴发和衰亡模拟试验, 3 个处理中各理化性 质随时间的变化情况见图 4. 由于实验初期添加了 $\mathrm{NH}_{4} \mathrm{Cl}$, Chl. a 浓度在前 3 天迅速上升, 与之相对应, $\mathrm{NH}_{4}^{+}-\mathrm{N}$ 浓度急剧下降, 在第 3 天下午所添加的 $\mathrm{NH}_{4}^{+}-\mathrm{N}$ 已经全部消耗, 而溶解氧浓度则急剧上升, 并呈现出明显的昼 夜变化. 第 4 天开始藻逐渐变黄, 而 Chl. a 浓度也呈现下降趋势, 表明藻开始衰亡, 至实验结束时各处理组中 Chl.a 浓度已下降至初始水平. 藻的衰亡降解需要消耗大量的溶解氧, 因此这个阶段 DO 开始下降, 到第 8 天 已下降至 $3.2 \mathrm{mg} / \mathrm{L}$. 由于有机氮的矿化需要一定的时间, 因此第 2 次添加的 $\mathrm{NH}_{4}^{+} \mathrm{-N}$ 继续被消耗, 直到第 $6 \sim 8$ 天才开始上升. 矿化作用为蓝藻的再暴发提供了一定量的营养元素, 从第 8 天开始蓝藻逐渐变绿, 而 DO 和 $\mathrm{pH}$ 也呈现上升趋势. 第 2 次添加 $\mathrm{NH}_{4}^{+}-\mathrm{N}$ 后, 不同处理中 $\mathrm{NH}_{4}^{+}-\mathrm{N}$ 浓度的变化差异显著 $(P<0.01, A N O V A)$, 具 体表现为初始藻浓度越高, $\mathrm{NH}_{4}^{+} \mathrm{N}$ 浓度越低, 其原因可能与蓝藻的同化作用和微生物硝化作用有关.

微生物的好氧降解能够迅速降低湖泊 CDOM 含量 ${ }^{[21]}$, 但是我们发现藻华暴发初期藻的同化作用和暴 发中后期微生物的反硝化作用在去除 CDOM 中同样有着巨大的贡献. 一般情况下, 蓝藻的光合作用会消耗 水中溶解的 $\mathrm{CO}_{2}$, 使得水体 $\mathrm{pH}$ 值升高. 但是许多研究表明, 蓝藻可以利用溶解性有机碳进行自身细胞的合 成 ${ }^{[22]}$, Silaban 等 ${ }^{[23]}$ 甚至发现添加有机碳后, 蓝藻的生长速率得到大大提高. 本研究中, 不同处理组中的 

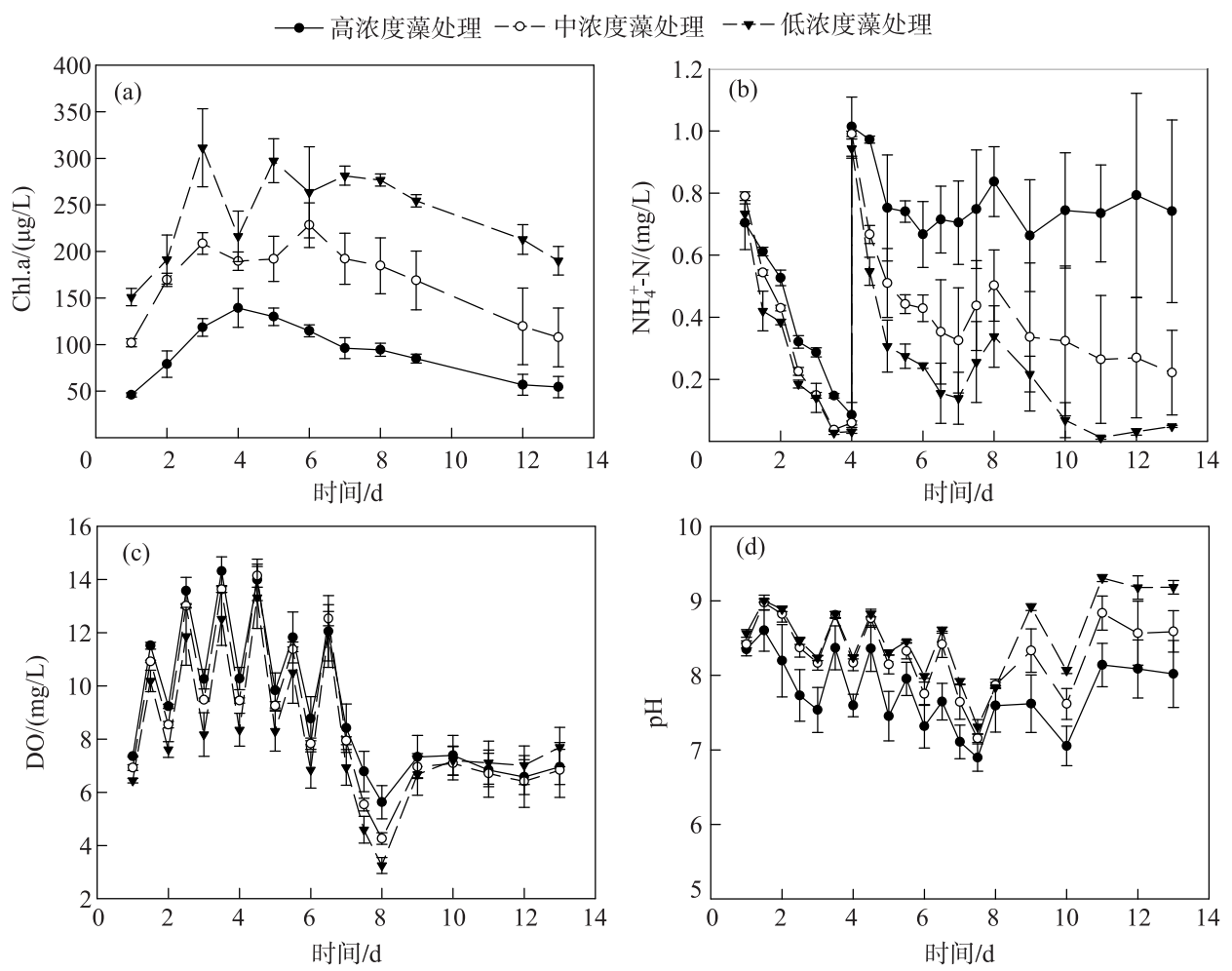

图 4 不同浓度藻的处理中 Chl.a( a )、 $\mathrm{NH}_{4}^{+}-\mathrm{N}(\mathrm{b}) 、 \mathrm{DO}(\mathrm{c})$ 和 $\mathrm{pH}(\mathrm{d})$ 随时间的变化

Fig.4 Temporal variations of Chl.a(a), $\mathrm{NH}_{4}^{+}-\mathrm{N}(\mathrm{b}), \mathrm{DO}(\mathrm{c})$ and $\mathrm{pH}(\mathrm{d})$ in different treatments

Chl.a 浓度均在前 3 天急剧上升, 而 $\mathrm{pH}$ 值仅在第 1 天少量上升, 到第 3 天才呈现出昼夜变化的趋势, 表明实 验初期蓝藻可能利用了水体中溶解性有机碳进行生长. 反硝化一般发生在 DO 浓度低于 $0.7 \mathrm{mg} / \mathrm{L}$ 的沉积层 与水柱庆氧界面处 ${ }^{[24]}$, 然而前期研究结果表明藻附生菌的反硝化能够在 DO 浓度较高的环境中发生 ${ }^{[25]}$. 实 验第 8 天 DO 已经下降至 $3.2 \mathrm{mg} / \mathrm{L}$, 而由于藻和微生物的呼吸作用, 夜间 DO 浓度可能会更低, 因此根据 $\mathrm{NO}_{3}^{-}-\mathrm{N}$ 浓度在第 2 次添加 $\mathrm{NH}_{4}^{+}-\mathrm{N}$ 后出现的白天上升、夜间下降的昼夜变化情况,结合不同处理中总氮浓度 的减少 (数据未列出), 可推测体系中存在着耦合硝化一反硝化作用, 从而消耗了水体中的溶解性有机物.

与太湖表层水体相似,模拟试验水样的三维苂光光谱同样解析出 3 个苂光组分 (图 5), 其中组分 1 (类 酪氨酸苂光物质) 的苂光峰位置分别为 $\mathrm{Ex} / \mathrm{Em}=225 \mathrm{~nm} / 320 \mathrm{~nm}$ 和 $\mathrm{Ex} / \mathrm{Em}=275 \mathrm{~nm} / 320 \mathrm{~nm}$, 组份 2 (类色氨 酸物质) 的荧光峰位置分别为 $E x / E m=235 \mathrm{~nm} / 345 \mathrm{~nm}$ 和 $E x / E m=300 \mathrm{~nm} / 345 \mathrm{~nm}$, 均与湖泊水样相应荧光 峰的位置基本一致. 组分 3 (类腐殖酸物质) 的 2 个荧光峰位置分别为 $\mathrm{Ex} / \mathrm{Em}=260 \mathrm{~nm} / 445 \mathrm{~nm}$ 和 $\mathrm{Ex} / \mathrm{Em}=$ $330 \mathrm{~nm} / 445 \mathrm{~nm}$, 与湖泊水样相比, 其激发波长和发射波长均有一定程度的蓝移 (图 3 和图 5), 相似的蓝移 现象在 Zhang 等 ${ }^{[12]}$ 的蓝藻降解试验中也曾被发现. 研究表明, 类腐殖酸苂光峰的发射波长与其芳香性显著 相关, 具体表现为芳香性越强, 发射波长越长 ${ }^{[26]}$. 湖泊外源 CDOM 主要来源于陆源植物和土壤有机质的分 解和淋溶, 其芳香性高于内源类腐殖质 ${ }^{[27]}$, 因此模拟试验中类腐殖酸物质苂光峰的蓝移可能是内源所占的 比例增大造成 ${ }^{[28]}$.

利用 PARAFAC 模型中的得分值计算 3 个组分对总苂光强度的贡献率 (图 6), 结果发现, 整个实验过程中 初始藻浓度越高, 类蛋白物质 (组分 1 和组分 2 ) 所占比例越低, 类腐殖酸物质 (组分 3$)$ 的比例越高 $(P<0.01$, ANOVA). 而添加了中等浓度和高浓度的藻处理中, 实验结束时类腐殖酸物质 (组分 3 ) 占总苂光物质的比例显 著高于实验初始时 $(P<0.01$, ANOVA $)$, 这证明了前文藻华暴发可以改变湖泊 CDOM 组成结构的推测.

在生长和衰亡阶段, 蓝藻可以为细菌提供大量的营养元素和躲避浮游动物滤食的庇护场所, 使得异养 

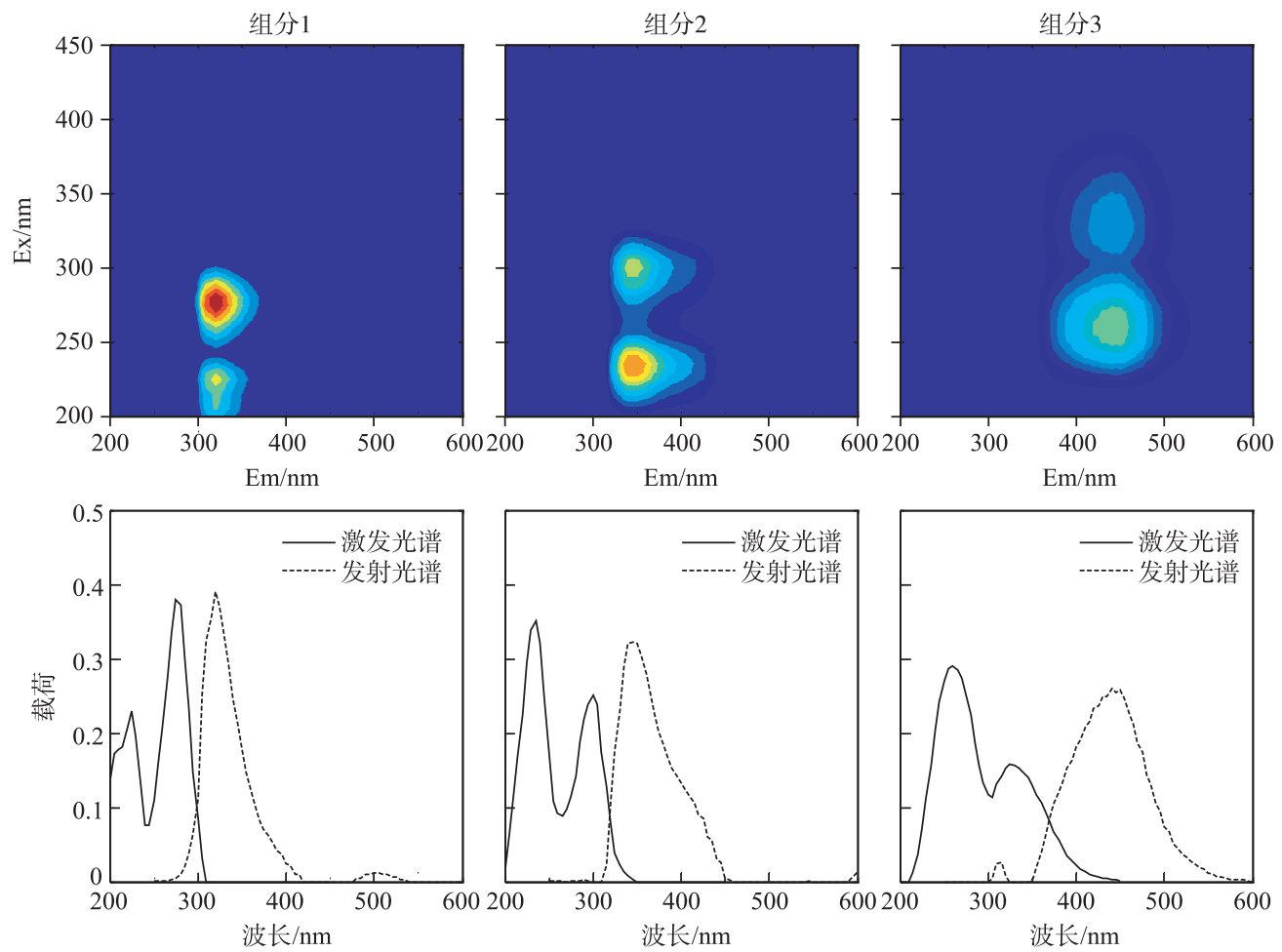

图 5 PARAFAC 模型解析出的 3 个组分

Fig.5 The PARAFAC model output showing fluorescence signatures of three components identified
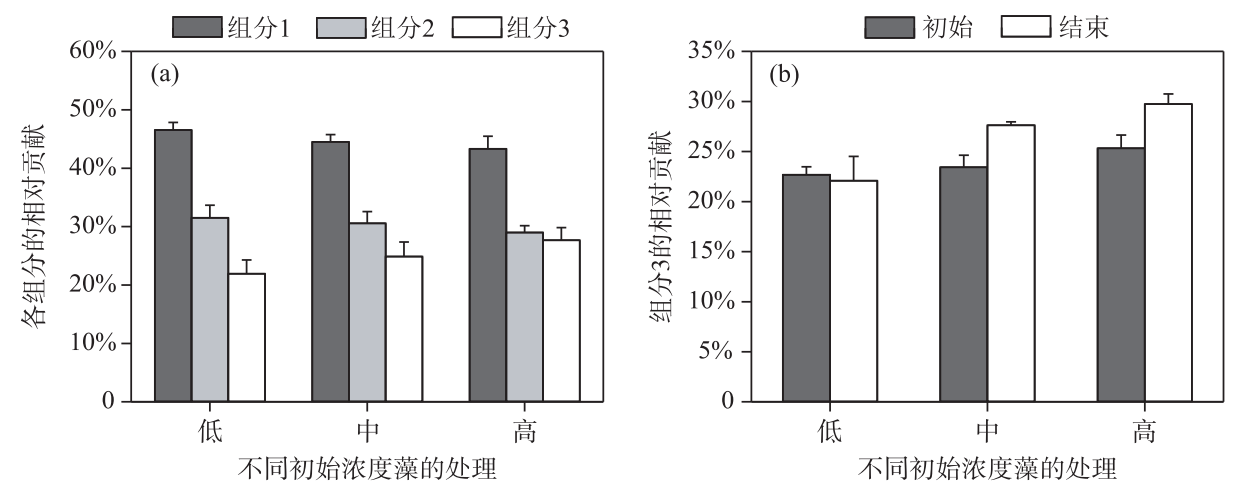

图 6 整个实验过程中各组分占总苂光物质的比例 (a) 和实验始末组分 3 占总苂光物质的比例 ( b)

Fig.6 Ratios of each component to total fluorescence substances during the experiment(a) and the initial and final ratios of component 3 to total fluorescence substances in different treatments (b)

型的藻附生菌和浮游细菌大大增殖 ${ }^{[29]}$. 研究表明, 这些异养型微生物在 CDOM 的代谢过程中具有双重作 用, 它们既是 CDOM 的生产者, 又是 CDOM 的消费者 ${ }^{[30]}$, 因此藻华暴发过程中急剧增殖的藻附生菌和浮游 细菌在促进 CDOM 产生的同时, 又大大加快了 CDOM 的微生物降解速率. 在富营养化程度较高的太湖西北 湖区, 夏季大量的蓝藻群体过度堆积, 导致水华下层容易出现蔽光的环境 ${ }^{[13]}$, 从而极大地削弱了湖泊 CDOM 的光降解效率. Stedmon 等 ${ }^{[31]}$ 发现, 浮游植物降解所产生的内源类腐殖质主要由光降解去除, 而类蛋白物质 
则同时受到光降解和微生物降解的作用. 因此, 藻华暴发和衰亡过程中, 藻的降解导致了类腐殖酸物质和类 蛋白物质含量的升高, 但是由于受藻华直接或间接的影响, CDOM 中类腐殖酸物质的降解速率远低于类蛋 白物质, 从而造成了类腐殖酸物质相对比例的升高. 由此可以推测, 蓝藻水华的长期暴发可能是除河道输人 外,梅梁湾类腐殖酸物质的比例高于开敞区的另一个重要原因.

\section{3 结论}

湖泊调查和模拟实验结果表明蓝藻是太湖 CDOM 的重要来源. 藻华的暴发可以改变湖泊 CDOM 的组 成, 促进类腐殖酸物质比例的升高. 蓝藻水华的长期暴发可能是除河道输人外, 梅梁湾类腐殖酸物质的比例 高于开敞区的另一个重要原因.

致谢: 感谢中国科学院太湖湖泊生态系统研究站为本研究提供研究场地和实验仪器设备的支持.

\section{4 参考文献}

[ 1 ] Arvola L, Äijälä C, Leppäranta M. CDOM concentrations of large Finnish lakes relative to their landscape properties. Hydrobiologia, 2016, 780 ( 1 ) : 37-46.

[ 2 ] Rochelle-Newall EJ, Fisher TR. Production of chromophoric dissolved organic matter fluorescence in marine and estuarine environments : an investigation into the role of phytoplankton. Marine Chemistry, 2002, 77(1) : 7-21.

[ 3 ] Zhou Y, Yao X, Zhang Y et al. Potential rainfall-intensity and $\mathrm{pH}$-driven shifts in the apparent fluorescent composition of dissolved organic matter in rainwater. Environmental Pollution, 2017, 224: 638-648.

[ 4 ] Sempéré R, Para J, Tedetti M et al. Variability of solar radiation and CDOM in surface coastal waters of the northwestern Mediterranean Sea. Photochemistry \& Photobiology, 2015, 91(4) : 851-861.

[ 5 ] Nelson NB, Siegel DA, Carlson CA et al. Tracing global biogeochemical cycles and meridional overturning circulation using chromophoric dissolved organic matter. Geophysical Research Letters, 2010, 37(3) : L03610.

[6] Zhang Y, Liu M, Qin B et al. Photochemical degradation of chromophoric-dissolved organic matter exposed to simulated UV-B and natural solar radiation. Hydrobiologia, 2009, 627(1) : 159-168.

[ 7 ] Coble PG. Characterization of marine and terrestrial DOM in seawater using excitation- emission matrix spectroscopy. Marine Chemistry, 1996, 51(4) : 325-346.

[ 8 ] Worms IAM, Adenmatten D, Miéville P et al. Photo-transformation of pedogenic humic acid and consequences for Cd ( II ), $\mathrm{Cu}($ II ) and $\mathrm{Pb}$ ( II ) speciation and bioavailability to green microalga. Chemosphere, 2015, 138: 908-915.

[ 9 ] Spencer RGM, Butler KD, Aiken GR. Dissolved organic carbon and chromophoric dissolved organic matter properties of rivers in the USA. Journal of Geophysical Research Biogeosciences, 2015, 117: G03001.

[10] Cammack WKL, Kalff J, Prairie YT et al. Fluorescent dissolved organic matter in lakes: Relationships with heterotrophic metabolism. Limnology and Oceanography, 2004, 49(6) : 2034-2045.

[11] Piccini C, Conde D, Pernthaler J et al. Alteration of chromophoric dissolved organic matter by solar UV radiation causes rapid changes in bacterial community composition. Photochemical \& Photobiological Sciences, 2009, 8( 9) : 1321-1328.

[12] Zhang Y, van Dijk MA, Liu M et al. The contribution of phytoplankton degradation to chromophoric dissolved organic matter( CDOM) in eutrophic shallow lakes: field and experimental evidence. Water Research, 2009, 43(18) : 4685-4697.

[13] Zhou Y, Jeppesen E, Zhang Y et al. Chromophoric dissolved organic matter of black waters in a highly eutrophic Chinese lake: Freshly produced from algal scums? Journal of Hazardous Materials, 2015, 299(6) : 222-230.

[14] Tfaily MM, Corbett JE, Wilson R et al. Utilization of PARAFAC-Modeled Excitation-Emission Matrix( EEM) Fluorescence Spectroscopy to identify biogeochemical processing of dissolved organic matter in a Northern Peatland. Photochemistry \& Photobiology, 2015, 91(3) : 684-695.

[15] Oloibiri V, De CS, Chys M et al. Characterisation of landfill leachate by EEM-PARAFAC-SOM during physical-chemical treatment by coagulation-flocculation, activated carbon adsorption and ion exchange. Chemosphere, 2017, 186: 873-883.

[16] Qin B ed. Lake Taihu, China: dynamics and environmental change. Springer Science \& Business Media, 2008.

[17] Guo L. Doing battle with the green monster of Taihu Lake. Science, 2007, 317(5842) : 1166-1166.

[18] Xu H, Paerl HW, Qin BQ et al. Nitrogen and phosphorus inputs control phytoplankton growth in eutrophic Lake Taihu, China. Limnology and Oceanography, 2010, 55(1): 420-432. 
[19] Ministry of Environmental Protection of the People's Republic of China, Editorial Board of Water and Wastewater Monitoring and Analysis Methods ed. Water and wastewater monitoring and analysis methods: 4th edition. Beijing: China Environmental Science Press, 2002. [ 国家环境保护总局《水和废水监测分析方法》编委会. 水和废水监测分析方法: 第 4 版. 北京: 中国环境科学出版社, 2002.]

[20] Hudson N, Baker A, Reynolds D. Fluorescence analysis of dissolved organic matter in natural, waste and polluted waters-A review. River Research and Applications, 2007, 23: 631-649.

[21] Hanamachi Y, Hama T, Yanai T. Decomposition process of organic matter derived from freshwater phytoplankton. Limnolo$g y, 2008, \mathbf{9}(1): 57-69$.

[22] Wan N, Abernathy M, Tang KH et al. Cyanobacterial photo-driven mixotrophic metabolism and its advantages for biosynthesis. Frontiers of Chemical Science and Engineering, 2015, 9(3): 308-316.

[23] Silaban A, Bai R, Gutierrez-Wing MT et al. Effect of organic carbon, C:N ratio and light on the growth and lipid productivity of microalgae/cyanobacteria coculture. Engineering in Life Sciences, 2014, 14(1) : 47-56.

[24] Brandes JA, Devol AH, Deutsch C. New developments in the marine nitrogen cycle. Chemical Reviews, 2007, 38(20): 577-589.

[25] Chen X, Jiang H, Sun X et al. Nitrification and denitrification by algae-attached and free-living microorganisms during a cyanobacterial bloom in Lake Taihu, a shallow Eutrophic Lake in China. Biogeochemistry, 2016, 131(1/2) : 135-146.

[26] Ateia M, Ran J, Fujii M et al. The relationship between molecular composition and fluorescence properties of humic substances. International Journal of Environmental Science \& Technology, 2017, 14(4) : 868-880.

[27] Mcknight DM, Boyer EW, Westerhoff PK et al. Spectrofluorometric characterization of dissolved organic matter for indication of precursor organic material and aromaticity. Limnology and Oceanography, 2001, 46(1) : 38-48.

[28] Boehme J, Wells M. Fluorescence variability of marine and terrestrial colloids: Examining size fractions of chromophoric dissolved organic matter in the Damariscotta River estuary. Marine Chemistry, 2006, 101(1/2) : 95-103.

[29] Niu Y, Shen H, Chen J et al. Phytoplankton community succession shaping bacterioplankton community composition in Lake Taihu, China. Water Research, 2011, 45(14) : 4169-4182.

[30] Guillemette F, del Giorgio PA. Simultaneous consumption and production of fluorescent dissolved organic matter by lake bacterioplankton. Environmental Microbiology, 2012, 14(6) : 1432-1443.

[31] Stedmon CA, Markager S. Tracing the production and degradation of autochthonous fractions of dissolved organic matter by fluorescence analysis. Limnology and Oceanography, 2005, 50(5) : 1415-1426. 